\title{
A Phase lb study of ruxolitinib + gemcitabine \pm nab-paclitaxel in patients with advanced solid tumors
}

This article was published in the following Dove Press journal:

OncoTargets and Therapy

\author{
Todd M Bauer ${ }^{1,2}$ \\ Manish R Patel ${ }^{1,3}$ \\ Andres Forero-Torres ${ }^{4}$ \\ Thomas J George Jr ${ }^{5}$ \\ Albert Assad ${ }^{6}$ \\ Yining $\mathrm{Du}^{6}$ \\ Herbert Hurwitz ${ }^{7}$ \\ 'Medical Oncology, Sarah Cannon \\ Research Institute, Nashville, TN, \\ USA; ${ }^{2}$ Medical Oncology, Tennessee \\ Oncology, Nashville, TN, USA; \\ ${ }^{3}$ Medical Oncology, Florida Cancer \\ Specialists, Sarasota, FL, USA; \\ ${ }^{4}$ Hematology and Oncology Division, \\ University of Alabama at Birmingham, \\ Birmingham, AL, USA; ${ }^{5}$ Department \\ of Medicine, University of Florida, \\ Gainesville, FL, USA; ${ }^{6}$ Biostatistics, \\ Incyte Corporation, Wilmington, DE, \\ USA; ${ }^{7}$ Department of Medicine, Duke \\ University Medical Center, Durham, \\ NC, USA
}

Purpose: Aberrant activation of the Janus-associated kinase (JAK)/signal transducer and activator of transcription (STAT) pathway is associated with increased malignant cell proliferation and survival. This Phase Ib study evaluated ruxolitinib, a potent JAK1/2 inhibitor, in combination with gemcitabine with or without nab-paclitaxel in patients with advanced solid tumors.

Patients and methods: Patients received ruxolitinib + gemcitabine (regimen A) or ruxolitinib + gemcitabine + nab-paclitaxel (regimen B). The objective of the dose-finding phase was to identify the maximum tolerated doses (MTDs) of ruxolitinib plus gemcitabine with or without nab-paclitaxel.

Results: Among 42 patients enrolled, the median age was 62.5 years, $81.0 \%$ had pancreatic cancer, and almost $62 \%$ had received prior systemic therapy. Regimen A was tolerated with standard doses of gemcitabine; regimen B was tolerated with reduced doses of gemcitabine/ nab-paclitaxel or concomitant granulocyte colony-stimulating factor. The sponsor decided to terminate the study early due to the interim analysis results of the Phase III JANUS 1 study. Discontinuations were mainly due to radiologic or clinical disease progression $(81.0 \%$ of patients). Median treatment durations were 55.5 days (cohort A0) and 150.5 days (pooled B cohorts). Four patients (pooled B cohorts) had dose-limiting toxicities: grade 3 pneumonia $(n=1)$, grade 4 neutropenia $(n=1)$, and grade 4 thrombocytopenia $(n=2)$. The most common grade 3/4 hematologic adverse events (AEs) were anemia, thrombocytopenia, and neutropenia. Serious AEs occurring in $\geq 2$ patients in cohort $A 0$ or pooled $B$ cohorts were abdominal pain, sepsis (cohort A0), dehydration, anemia, and asthenia (pooled B cohorts). Overall response rates (ORRs) were $12.5 \%$ in cohort A0 and $38.5 \%$ in pooled B cohorts. Among patients with pancreatic cancer, ORR was $23.5 \%$ (14.0\% cohort A0 30.0\% pooled B cohorts).

Conclusion: The study was terminated early prior to reaching MTDs per sponsor decision; although ruxolitinib plus gemcitabine with or without nab-paclitaxel was generally safe and well tolerated in patients with advanced solid tumors, this combination will not be pursued further.

Keywords: Janus kinase inhibitor, pancreatic cancer

\section{Introduction}

The Janus-associated kinase (JAK) family comprises 4 non-receptor protein tyrosine kinases (JAK1-3 and tyrosine kinase 2 ) that transduce cytokine-mediated signaling via the JAK/signal transducer and activator of transcription (STAT) pathway. ${ }^{1}$ Aberrant activation of this pathway is associated with increased malignant cell proliferation and survival ${ }^{1,2}$ and has been observed in various tumor types. ${ }^{3-5}$ Furthermore, JAK kinases are key mediators of downstream signaling for various cytokine and/or growth factor
Correspondence: Todd M Bauer Research Institute, 250 25th Avenue North, Suite 200, Nashville, TN 37203, USA

Tel +l 6153297274

Fax + I 6I5 5244939

Email tbauer@tnonc.com 
receptors that play a role in systemic inflammation, leading to cancer cachexia that increases cancer-associated morbidity and mortality. ${ }^{1,6-8}$

Ruxolitinib, an orally bioavailable, potent, and selective inhibitor of JAK 1 and 2 enzymes, has been approved by the US Food and Drug Administration and European Medicines Agency for the treatment of intermediate- to high-risk primary or secondary myelofibrosis. ${ }^{9}{ }^{10}$ By selectively inhibiting JAK2 V617F, STAT5, and ERK1/2 phosphorylation, ruxolitinib reduces cellular proliferation and induces apoptosis of JAK2 V617F Ba/F3 cells. ${ }^{11}$ Ruxolitinib has also been shown to possess activity in solid tumors. In a previous Phase II study of Ruxolitinib in Pancreatic Cancer Patients (RECAP), early evidence of ruxolitinib in combination with capecitabine suggested an association with improved survival compared with placebo and capecitabine in a subset of patients with metastatic pancreatic cancer and systemic inflammation, evidenced by elevated C-reactive protein levels. ${ }^{12}$

In addition to approvals in other solid tumor settings, gemcitabine is indicated as a single agent for pancreatic cancer and in combination with nab-paclitaxel as a first-line treatment for metastatic adenocarcinoma of the pancreas; ${ }^{13,14}$ however, myelosuppression is a common and sometimes dose-limiting adverse event (AE) associated with both gemcitabine and nab-paclitaxel. ${ }^{13,14}$ Ruxolitinib has demonstrated the ability to reduce levels of pro-inflammatory cytokines (thought to be associated with the development of cachexia) in patients with myelofibrosis. ${ }^{15-17}$ Ruxolitinib is generally known to be well tolerated and may augment the activity of gemcitabine in patients with pancreatic cancer. Indeed, in a murine model of pancreatic cancer, the combination of gemcitabine plus a JAK2 inhibitor (fedratinib) substantially reduced the rate of tumor growth and significantly improved overall survival compared with either agent alone. ${ }^{18}$ Overlapping hematologic toxicities associated with gemcitabine and ruxolitinib may be a concern clinically.

The current Phase Ib, dose-finding study was conducted to evaluate the safety and tolerability of ruxolitinib in combination with gemcitabine with or without nab-paclitaxel in patients with advanced pancreatic cancer and other advanced solid tumors. Our study was terminated early by the sponsor after an interim analysis from a Phase III trial of ruxolitinib plus capecitabine (JANUS 1) showed no additional benefit over capecitabine alone in patients with advanced pancreatic cancer and high systemic inflammation as measured by the modified Glasgow prognostic score. ${ }^{19}$

\section{Patients and methods}

\section{Patients}

Eligible patients were aged $\geq 18$ years, had radiographically measurable advanced or metastatic pancreatic adenocarcinoma or another advanced solid tumor, and an Eastern Cooperative Oncology Group (ECOG) performance status of $\leq 1$.

The study was conducted in accordance with the study protocol, Declaration of Helsinki, Good Clinical Practices as defined in the Code of Federal Regulations Title 21, International Conference of Harmonisation - Good Clinical Practice consolidated guidelines, and applicable regulatory requirements. All patients provided written informed consent before study participation. The study protocol and its amendments and patients' informed consent were reviewed and approved by institutional review boards or independent ethics committees (IntegReview Ethical Review Board, Austin, TX, USA; Western Institutional Review Board, Puyallup, WA, USA; Western Institutional Review Board, Olympia, WA, USA; and Duke University Health System Institutional Review Board, Durham, NC, USA).

\section{Study design}

The Phase Ib open-label study (NCT01822756) was designed to be conducted in 2 parts. Part 1 comprised a dose-finding phase to identify the maximum tolerated dose (MTD) of ruxolitinib when administered with gemcitabine with or without nab-paclitaxel to patients with advanced or metastatic pancreatic cancer and other advanced solid tumors; safety, tolerability, and pharmacokinetics would be assessed in part 1. Part 2 was to explore the safety, tolerability, pharmacokinetics, pharmacodynamics, and preliminary clinical activity of ruxolitinib, at the dose identified in part 1 , in combination with gemcitabine with or without nab-paclitaxel. Part 2 of the study was not conducted following a sponsor decision not to pursue the combinations of ruxolitinib + gemcitabine or ruxolitinib + gemcitabine/nab-paclitaxel, and therefore, to stop further enrollment during part 1 before the MTD was reached.

Part 1 was a $3+3$ dose-finding design in which patients were assigned to 1 of 2 treatment regimens, regimen A or B, based on prior chemotherapy exposure (Figure 1). Patients who had received no more than 1 prior chemotherapy regimen for advanced or metastatic disease (excluding neoadjuvant or adjuvant disease) were eligible to receive ruxolitinib + gemcitabine (regimen A). Patients who had not received prior chemotherapy for advanced or metastatic disease (excluding 

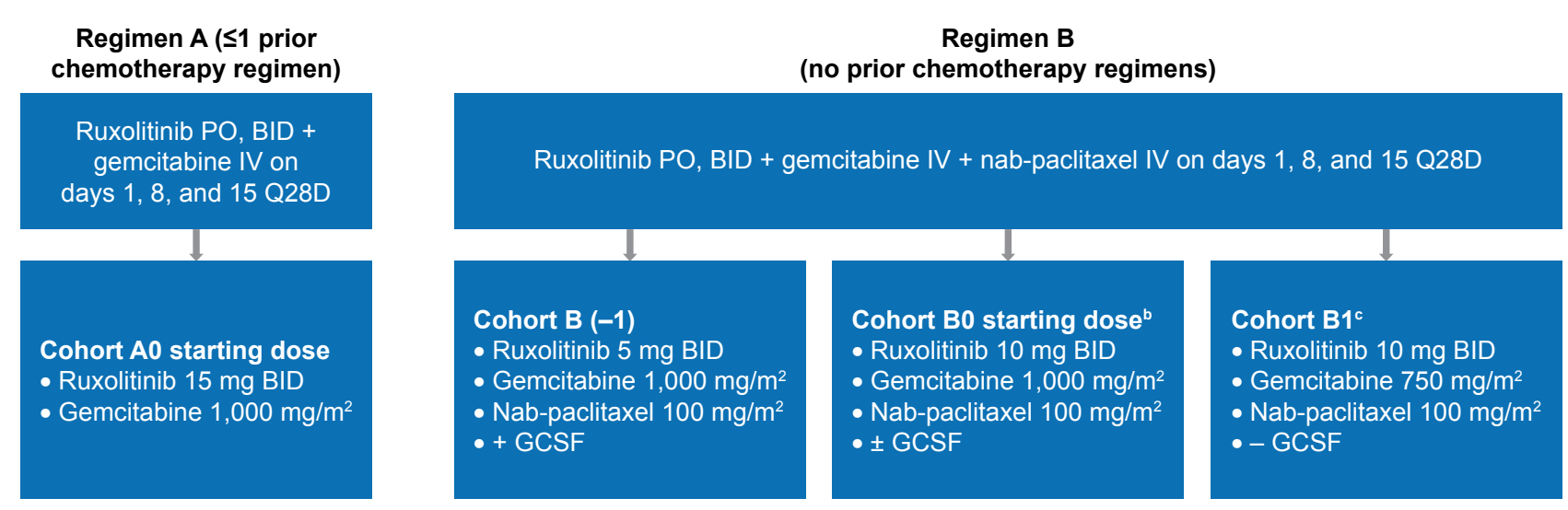

Figure I Study design. ${ }^{\mathrm{a}}$

Notes: aRepresents part I study design only. Part 2 (dose expansion at the maximum tolerated dose to be defined in part I) was not conducted. ${ }^{\text {b}}$ Cohort B0 was first tested without GCSF but was not tolerated; therefore, the cohort was again tested with GCSF. clf cohort B0 was not tolerated, cohort BI was tested to assess if, by lowering gemcitabine dose, the regimen was tolerated without GCSF.

Abbreviations: BID, twice daily; GCSF, granulocyte colony-stimulating factor; IV, intravenous; PO, orally; Q28D, every 28 days.

neoadjuvant or adjuvant disease) were eligible to receive ruxolitinib + gemcitabine + nab-paclitaxel (regimen B).

The first patients enrolled to regimen A were assigned to the starting dose cohort A0, consisting of ruxolitinib $15 \mathrm{mg}$ twice daily (BID) and gemcitabine $1,000 \mathrm{mg} / \mathrm{m}^{2}$ on days 1,8 , and 15 every 28 days (Q28D). The first patients enrolled to regimen $\mathrm{B}$ were assigned to the starting dose cohort B0, consisting of ruxolitinib $10 \mathrm{mg} \mathrm{BID}$, gemcitabine $1,000 \mathrm{mg} / \mathrm{m}^{2}$, and nab-paclitaxel $100 \mathrm{mg} / \mathrm{m}^{2}$ on days 1,8 , and 15 Q28D. Cohort B0 administered with prophylactic granulocyte colony-stimulating factor (GCSF) support could be repeated in patients with pancreatic cancer if any of the dose-limiting toxicities (DLTs) observed were due to myelosuppression. If a DLT was observed in 1 patient, then that cohort was expanded to at least 6 patients. If no additional DLTs were observed in those 3 additional patients, then dose finding could proceed or result in cohort expansion. If a DLT occurred in 2 patients or more of the total cohort, then the MTD was deemed to be exceeded, and the lower dose level was to be expanded to at least 6 patients to evaluate safety. If the regimen in cohort B0 was not tolerated, then an additional cohort (B1) was added to test a reduced dose of gemcitabine at $750 \mathrm{mg} / \mathrm{m}^{2}$, without the addition of prophylactic GCSF support. In addition, ruxolitinib $5 \mathrm{mg}$ BID, gemcitabine $1,000 \mathrm{mg} / \mathrm{m}^{2}$, and nab-paclitaxel $100 \mathrm{mg} / \mathrm{m}^{2}$ on days 1,8 , and 15 Q28D were tested in cohort B (-1) to evaluate the effects of GCSF with the expectation of re-escalating doses.

Treatment for all patients in regimens A and B consisted of repeating 28-day cycles, with treatment cycles continuing for as long as the regimen was tolerated and the patient did not meet discontinuation criteria. The expected duration was $\sim 4-6$ months.

\section{End points}

The primary end points were the determination of the MTD of ruxolitinib in combination with gemcitabine with or without nab-paclitaxel, and the safety and tolerability of the treatment regimens. Secondary end points were pharmacokinetics and pharmacodynamics. Because of the early termination of the study, samples for pharmacokinetics and pharmacodynamics, and computed tomography for tumor burden were collected but not analyzed. The exploratory end point was antitumor effects assessed as overall response rate (ORR).

\section{Assessments and statistical methods}

Safety and tolerability were assessed by monitoring AEs, measuring vital signs and 12-lead electrocardiograms, and evaluating physical examinations and clinical laboratory blood tests. The severity of AEs was graded using the National Cancer Institute Common Terminology Criteria for AEs v4.03. Safety data were compared over time to assess change from baseline, during treatment, and follow-up. The safety population, including all enrolled patients who received at least 1 dose of ruxolitinib (treatment group defined according to actual treatment received regardless of assigned study drug treatment), was used for all safety analyses. Response was determined by radiographic disease assessments per Response Evaluation Criteria in Solid Tumors version $1.1^{20}$ by investigator assessment and was exploratory in nature with descriptive statistics (eg, mean, standard deviation, and range) provided. The intent-to-treat 
(ITT) population, including all patients who received at least 1 dose of ruxolitinib (treatment group defined according to assigned treatment regardless of actual study drug received), was used for all efficacy analyses.

\section{Results}

\section{Patients}

A total of 42 patients with advanced solid tumors (predominantly pancreatic cancer; $81.0 \%$ ) were enrolled in part 1 of the study (ITT and safety population; Table 1). The median age at the time of enrollment was 62.5 years, and all patients had an ECOG performance status of either $0(57.1 \%)$ or $1(42.9 \%)$ at baseline. Almost $62 \%$ of patients had received prior systemic therapy for their primary cancer; $\sim 19 \%$ had received prior gemcitabine; and $\sim 40 \%$ had received prior radiation therapy. Of the 16 patients who had received prior radiation therapy, 8 received radiation therapy at the same time as chemotherapy as an add-on, 3 received neoadjuvant radiation therapy, and 5 received adjuvant radiation therapy.

\section{Exposure, safety, and efficacy MTD and exposure}

Ruxolitinib + gemcitabine (regimen A) was tolerated with standard doses of gemcitabine $\left(1,000 \mathrm{mg} / \mathrm{m}^{2}\right)$. Ruxolitinib + gemcitabine/nab-paclitaxel (regimen B) was tolerated with reduced doses of gemcitabine $\left(750 \mathrm{mg} / \mathrm{m}^{2}\right) / \mathrm{nab}$-paclitaxel $\left(100 \mathrm{mg} / \mathrm{m}^{2}\right)$ or with concomitant GCSF. MTDs were not defined, as the study was terminated after cohort B1 when the sponsor decided not to pursue these combinations further based on the interim results from another Phase III trial.

A total of 40 patients $(95.2 \%)$ discontinued treatment, primarily due to radiologic or clinical disease progression ( $n=34$ [81.0\%]; Table 2). Other reasons included AE (7.1\%), death $(4.8 \%)$, and patient decision $(2.4 \%)$. Overall, the median duration of ruxolitinib exposure was 97.5 days (55.5 days in cohort A0; 150.5 days in the pooled B cohorts); a median of 8.5 (range, 1-46) intravenous gemcitabine treatments were administered.

\section{Safety}

There were no DLTs experienced in cohort A0. Four patients in the B0 cohorts experienced DLTs; 2 patients from cohort B0 without GCSF, including 1 case of grade 4 neutropenia, 1 case of grade 4 thrombocytopenia, and 2 patients from cohort B0 with GCSF, including a serious event of grade 3 pneumonia (which resolved with treatment 6 days after onset) and 1 case of grade 4 thrombocytopenia.
All patients experienced at least 1 treatment-emergent AE (TEAE), with the most common nonhematologic TEAEs of any grade (occurring in $\geq 50 \%$ of patients in cohort $A$ or pooled B cohorts) being fatigue, nausea, alopecia, and peripheral edema (Table 3). A lower percentage of infections and infestations occurred in patients who did not receive GCSF compared with patients who did receive GCSF (28.6\% vs 57.1\%). Grade 3/4 TEAEs were reported in $>80 \%$ of patients in both cohort $\mathrm{A} 0$ and pooled $\mathrm{B}$ cohorts. The most common grade $3 / 4$ nonhematologic AEs (occurring in $\geq 10 \%$ of patients in cohort A or pooled B cohorts) were abdominal pain, fatigue, sepsis, and hypokalemia (Table 3); the most common grade 3/4 hematologic AEs (new/worsening laboratory abnormalities occurring in $\geq 10 \%$ of patients in cohort A or pooled B cohorts) were anemia, thrombocytopenia, and neutropenia (Table 4).

Treatment-emergent serious AEs (SAEs) were experienced in $62.5 \%$ of patients in cohort $\mathrm{A}$ and $34.6 \%$ of patients in pooled B cohorts. Treatment-emergent SAEs occurring in $\geq 2$ patients in either cohort A or pooled B cohorts were abdominal pain ( $25 \%$ cohort A0), sepsis ( $12.5 \%$ cohort A0), dehydration, anemia, and asthenia $(7.7 \%$ each, pooled $B$ cohorts). One death occurred in cohort A0 due to sepsis (without neutropenia), which was not considered related to ruxolitinib; no deaths occurred in pooled B cohorts.

\section{Efficacy}

ORRs were $12.5 \%(2 / 16)$ in cohort A0 and 38.5\% (10/26) in the pooled B cohorts (Table 5). Among the patients with pancreatic cancer, ORR was $23.5 \%$ (14.0\% in cohort A0 and $30.0 \%$ in pooled $B$ cohorts). A complete response (CR) occurred in 1 patient $(2.4 \%)$ overall with non-small cell lung cancer in cohort B0 without GCSF, and a partial response (PR) occurred in 11 patients overall (26.2\%). Among patients who achieved a PR, the median duration of response ranged from 33.0 days in cohort B (-1) to 225.0 days in cohort B1. The majority of PRs (82.0\%) occurred in patients treated with ruxolitinib for a median of $>140$ days. Clinical benefit rate (ORR + stable disease for $\geq 7$ weeks) was $37.5 \%(6 / 16)$ in cohort A0 and 73.0\% (19/26) for the pooled B cohorts. The largest percentage reduction of target lesions occurred in a patient with PR in cohort B (-1) and in a patient with $\mathrm{CR}$ in cohort B0 (Figure 2).

\section{Discussion}

In patients with chemotherapy-naive metastatic pancreatic adenocarcinoma, the combination of gemcitabine and nabpaclitaxel has previously demonstrated improved survival 


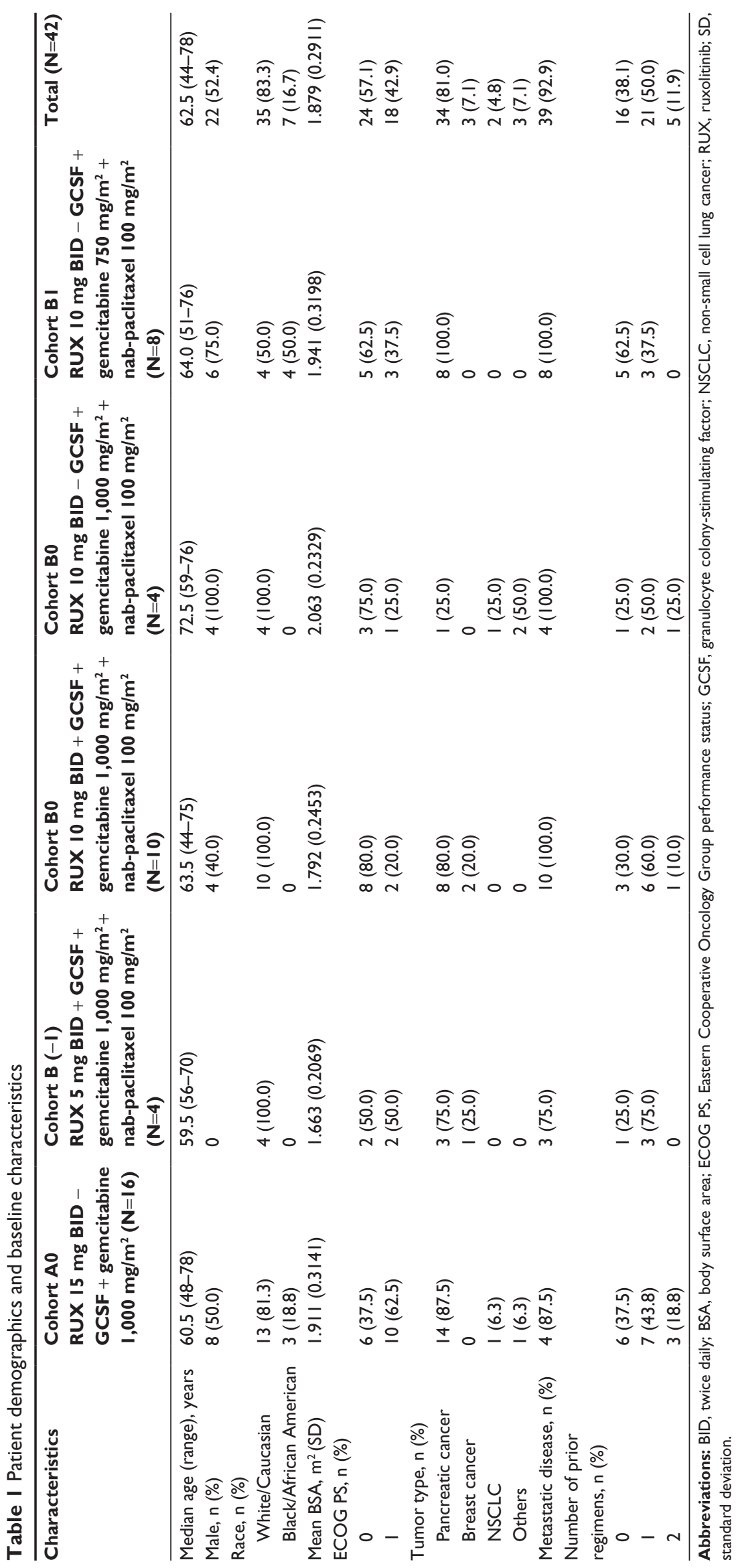



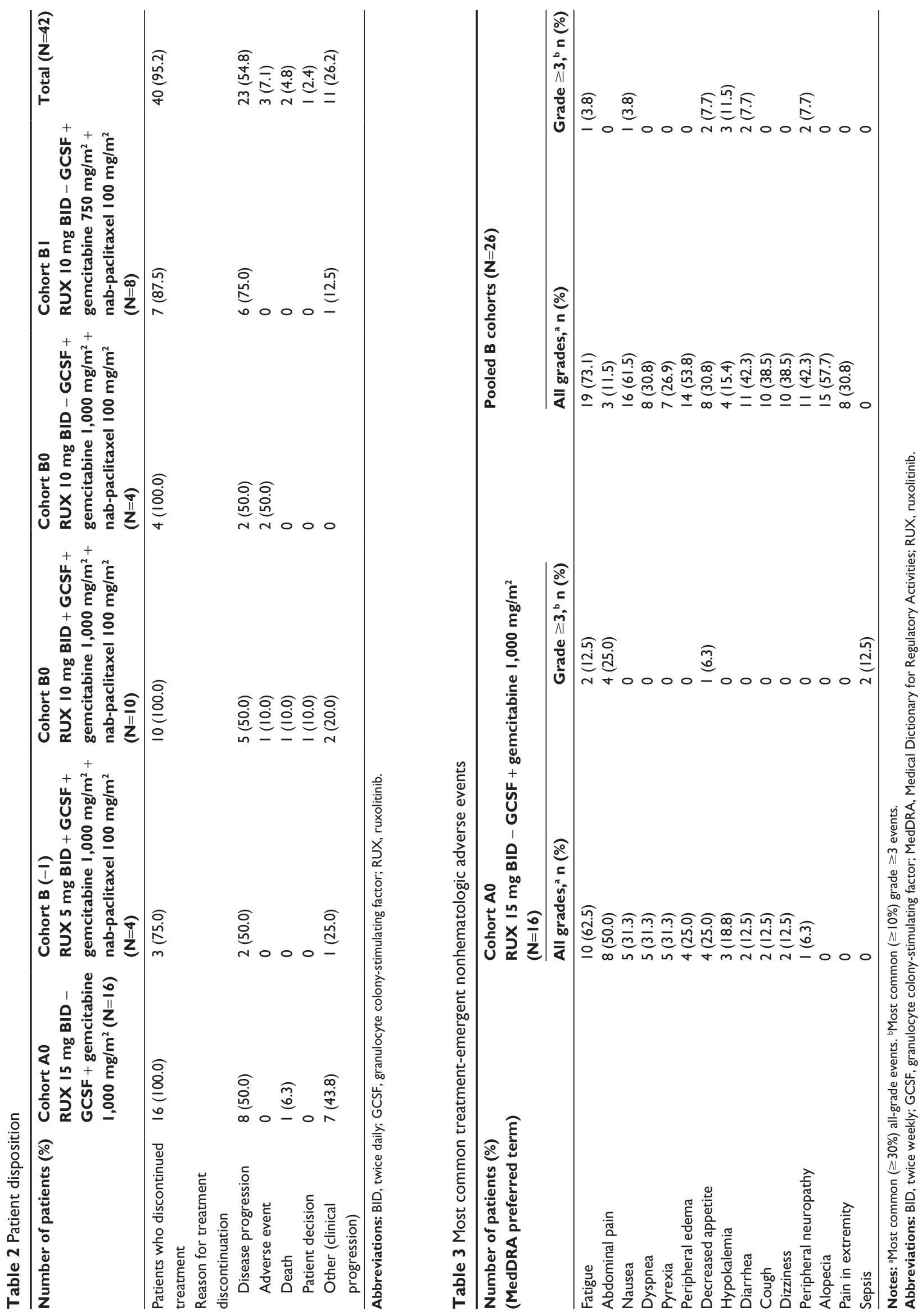

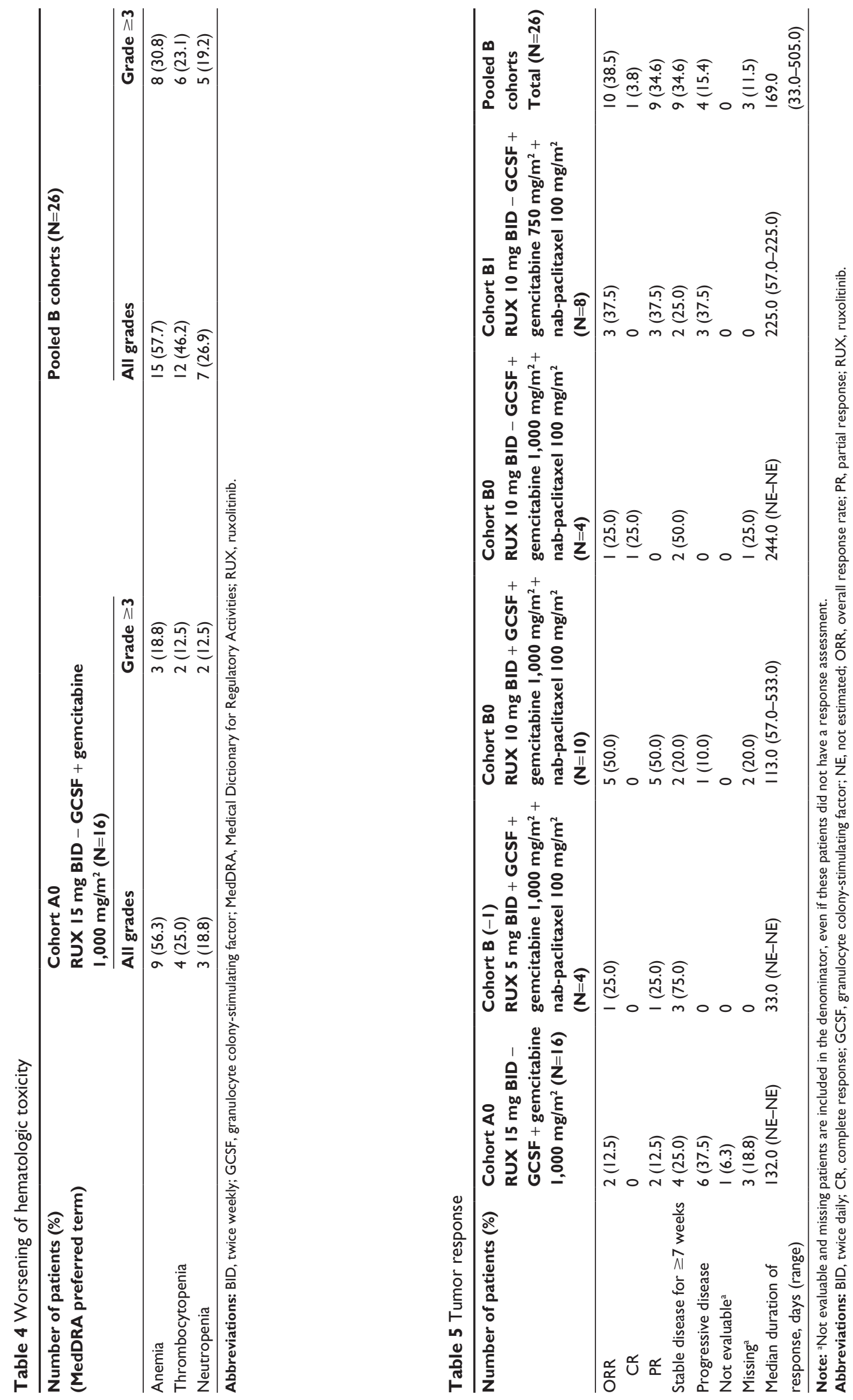


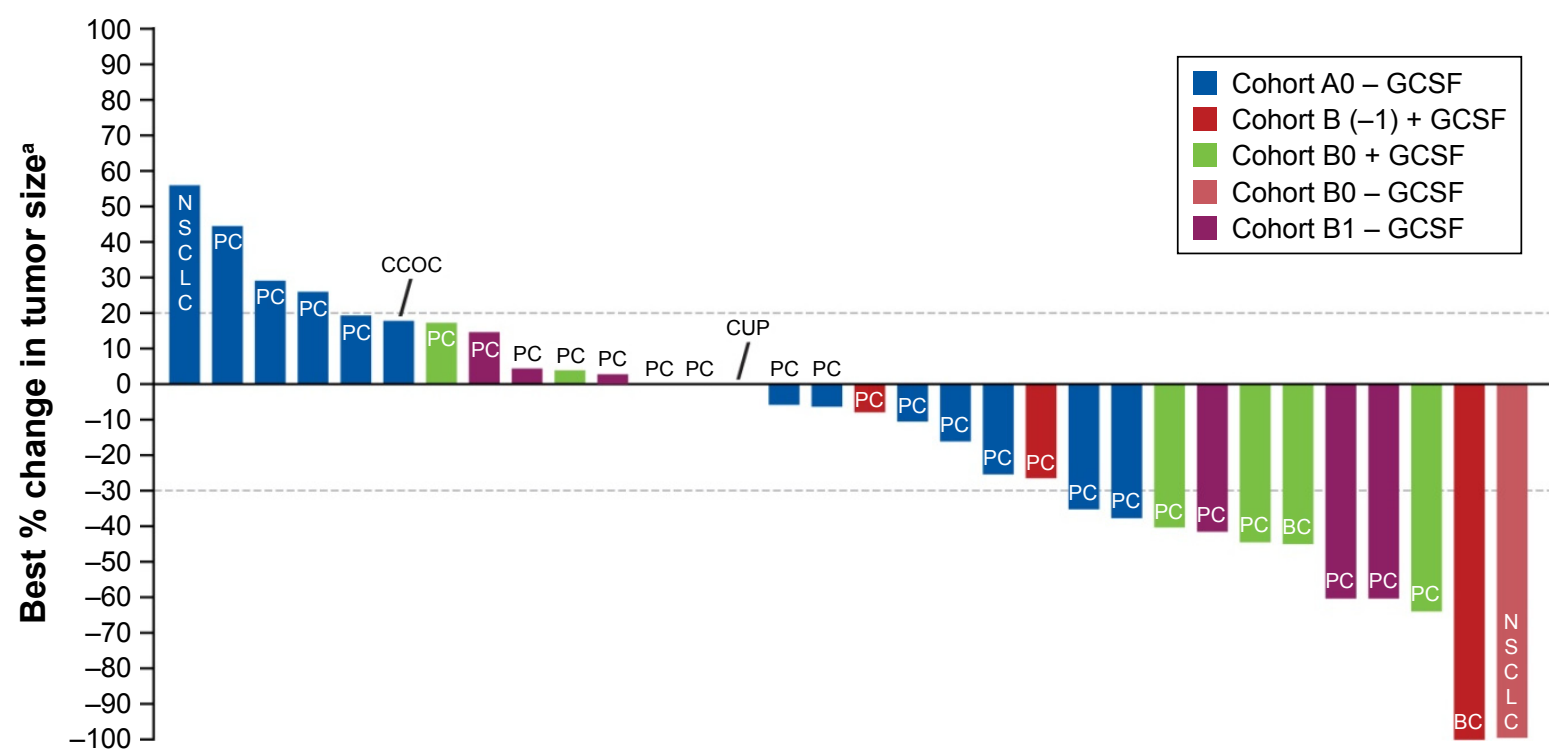

\section{Patients}

Figure 2 Waterfall plot of best percent change in sum of target lesions (ITT population).

Note: ${ }^{\mathrm{F}} \mathrm{For}$ patients who only had increases in target lesion size from baseline, the smallest increase was considered as the best change from baseline.

Abbreviations: BC, breast cancer; CCOC, clear cell odontogenic cancer; CUP, cancer of unknown primary (likely gallbladder); GCSF, granulocyte colony-stimulating factor; ITT, intent-to-treat; NSCLC, non-small cell lung cancer; PC, pancreatic cancer.

and tumor response compared with gemcitabine alone; however, this combination was also found to increase the rates of peripheral neuropathy and myelosuppression observed. ${ }^{21,22}$ In the Phase II RECAP study in patients with metastatic pancreatic cancer who had failed prior gemcitabine therapy, the combination of ruxolitinib and capecitabine demonstrated clinical activity, especially in patients with systemic inflammation, evidenced by elevated C-reactive protein levels. ${ }^{12}$ These results suggest that the modulation of inflammatory cytokine signaling may be important in these patients and highlight a potential role for JAK inhibition as a therapeutic target. ${ }^{12}$

The combination of ruxolitinib and gemcitabine, with and without nab-paclitaxel, in patients with advanced solid tumors, $81 \%$ of whom had pancreatic cancer, at the doses investigated were generally safe and well tolerated. The combination of ruxolitinib and gemcitabine was tolerated with standard doses of gemcitabine; however, the combination of ruxolitinib, gemcitabine, and nab-paclitaxel was tolerated with reduced doses of gemcitabine/nab-paclitaxel or with concomitant GCSF. Dose reductions of gemcitabine and/or nab-paclitaxel were required in up to $47 \%$ of patients in the pivotal study of gemcitabine versus gemcitabine plus nab-paclitaxel in patients with metastatic pancreatic cancer, and $15 \%-26 \%$ of patients received growth factor support. ${ }^{22}$ Although the overall number of patients treated was relatively small and any results from a comparison between groups must be interpreted with caution, the addition of GCSF to the ruxolitinib combination suggested no apparent clinical advantage, as a higher percentage of patients in cohorts receiving GCSF reported more infections than cohorts who did not receive GCSF. The most frequently reported TEAE was fatigue, which occurred in at least $50 \%$ of patients across all cohorts, and the most frequently reported grade $\geq 3$ TEAEs were anemia, thrombocytopenia, and neutropenia, consistent with the prescribing information for ruxolitinib. ${ }^{9,10}$ In 2 randomized double-blind Phase III trials, JANUS 1 and JANUS 2, ruxolitinib, when combined with capecitabine, also had a reasonably well-tolerated safety profile in patients with refractory advanced/metastatic pancreatic cancer. However, the addition of ruxolitinib to capecitabine did not improve clinical or quality of life outcomes, and the studies were terminated early based on efficacy findings of the planned interim analysis of JANUS $1 .{ }^{19}$

In our study, objective responses were noted in $23.5 \%$ of 34 patients with pancreatic cancer. These results correlate well with response rates determined from recent studies in patients with advanced/metastatic pancreatic cancer (7\%-35\%) depending on the treatment regimen used..$^{22-27}$

\section{Conclusion}

In this Phase I study of ruxolitinib in combination with gemcitabine with or without nab-paclitaxel in patients with advanced solid tumors, assessment of the safety data revealed that no new safety concerns were identified, and the doses investigated were generally well tolerated. The study was 
terminated early prior to reaching the MTDs per sponsor decision. The combinations of ruxolitinib and gemcitabine or ruxolitinib, gemcitabine, and nab-paclitaxel will not be pursued further.

\section{Acknowledgments}

This study was supported by Incyte Corporation. Medical writing assistance was provided by Jordana Campbell, part of Evidence Scientific Solutions, Inc, Philadelphia, PA, USA, and was funded by Incyte Corporation. AF-T has received research funding from Incyte (Inst) and Celgene (Inst). TJG Jr is a consultant for Merck and Bayer. AA has received research funding and royalties from Incyte. $\mathrm{HH}$ has received research funding from Incyte (Inst).

\section{Disclosure}

Both AA and YD are employees of Incyte Corporation and have stock ownership. The authors report no other conflicts of interest in this work.

\section{References}

1. Thomas SJ, Snowden JA, Zeidler MP, Danson SJ. The role of JAK/ STAT signalling in the pathogenesis, prognosis and treatment of solid tumours. Br J Cancer. 2015;113(3):365-371.

2. Quintás-Cardama A, Kantarjian H, Cortes J, Verstovsek S. Janus kinase inhibitors for the treatment of myeloproliferative neoplasias and beyond. Nat Rev Drug Discov. 2011;10(2):127-140.

3. Cortas T, Eisenberg R, Fu P, Kern J, Patrick L, Dowlati A. Activation state EGFR and STAT-3 as prognostic markers in resected non-small cell lung cancer. Lung Cancer. 2007;55(3):349-355.

4. Komazaki T, Nagai H, Emi M, et al. Hypermethylation-associated inactivation of the SOCS-1 gene, a JAK/STAT inhibitor, in human pancreatic cancers. Jpn J Clin Oncol. 2004;34(4):191-194.

5. Tam L, McGlynn LM, Traynor P, Mukherjee R, Bartlett JM, Edwards J. Expression levels of the JAK/STAT pathway in the transition from hormone-sensitive to hormone-refractory prostate cancer. Br J Cancer. 2007;97(3):378-383.

6. Carson JA, Baltgalvis KA. Interleukin 6 as a key regulator of muscle mass during cachexia. Exerc Sport Sci Rev. 2010;38(4):168-176.

7. Evans WJ, Morley JE, Argilés J, et al. Cachexia: a new definition. Clin Nutr. 2008;27(6):793-799.

8. Mondello P, Lacquaniti A, Mondello S, et al. Emerging markers of cachexia predict survival in cancer patients. BMC Cancer. 2014; $14: 828$.

9. JAKAFI ${ }^{\circledR}$ (ruxolitinib) [prescribing information]. Wilmington, DE, USA: Incyte Corporation; 2016.

10. JAKAVI ${ }^{\circledR}$ (ruxolitinib) [summary of product characteristics]. Camberley, UK: Novartis Europharm Limited; 2016.

OncoTargets and Therapy

\section{Publish your work in this journal}

OncoTargets and Therapy is an international, peer-reviewed, open access journal focusing on the pathological basis of all cancers, potential targets for therapy and treatment protocols employed to improve the management of cancer patients. The journal also focuses on the impact of management programs and new therapeutic agents and protocols on
11. Quintás-Cardama A, Vaddi K, Liu P, et al. Preclinical characterization of the selective JAK1/2 inhibitor INCB018424: therapeutic implications for the treatment of myeloproliferative neoplasms. Blood. 2010;115(15):3109-3117.

12. Hurwitz HI, Uppal N, Wagner SA, et al. Randomized, double-blind, phase II study of ruxolitinib or placebo in combination with capecitabine in patients with metastatic pancreatic cancer for whom therapy with gemcitabine has failed. J Clin Oncol. 2015;33(34):4039-4047.

13. ABRAXANE ${ }^{\circledR}$ (nab-paclitaxel) [prescribing information]. Summit, NJ, USA: Celgene Corporation; 2015.

14. GEMZAR ${ }^{\circledR}$ (gemcitabine) [prescribing information]. Indianapolis, IN, USA: Lilly USA; 2017.

15. Harrison C, Kiladjian JJ, Al-Ali HK, et al. JAK inhibition with ruxolitinib versus best available therapy for myelofibrosis. $N$ Engl $J$ Med. 2012;366(9):787-798.

16. Verstovsek S, Kantarjian H, Mesa RA, et al. Safety and efficacy of INCB018424, a JAK1 and JAK2 inhibitor, in myelofibrosis. $N$ Engl J Med. 2010;363(12):1117-1127.

17. Verstovsek S, Mesa RA, Gotlib J, et al. A double-blind, placebocontrolled trial of ruxolitinib for myelofibrosis. N Engl J Med. 2012; 366(9):799-807.

18. Wormann SM, Song L, Ai J, et al. Loss of P53 function activates JAK2-STAT3 signaling to promote pancreatic tumor growth, stroma modification, and gemcitabine resistance in mice and is associated with patient survival. Gastroenterology. 2016;151(1):180-193.e12.

19. Hurwitz H, Van Cutsem E, Bendell JC, et al. Two randomized, placebo-controlled phase 3 studies of ruxolitinib (Rux) + capecitabine (C) in patients (pts) with advanced/metastatic pancreatic cancer (mPC) after failure/intolerance of first-line chemotherapy: JANUS 1 (J1) and JANUS 2 (J2). J Clin Oncol. 2017;35(4 Suppl):343.

20. Eisenhauer EA, Therasse P, Bogaerts J, et al. New response evaluation criteria in solid tumours: revised RECIST guideline (version 1.1). Eur J Cancer. 2009;45(2):228-247.

21. Goldstein D, El-Maraghi RH, Hammel P, et al. nab-Paclitaxel plus gemcitabine for metastatic pancreatic cancer: long-term survival from a phase III trial. $J$ Natl Cancer Inst. 2015;107(2):dju413.

22. Von Hoff DD, Ervin T, Arena FP, et al. Increased survival in pancreatic cancer with nab-paclitaxel plus gemcitabine. $N$ Engl J Med. 2013;369(18):1691-1703.

23. Bendell J, O'Reilly EM, Middleton MR, et al. Phase I study of olaparib plus gemcitabine in patients with advanced solid tumours and comparison with gemcitabine alone in patients with locally advanced/metastatic pancreatic cancer. Ann Oncol. 2015;26(4):804-811.

24. Ikeda M, Sato A, Mochizuki N, et al. Phase I trial of GBS-01 for advanced pancreatic cancer refractory to gemcitabine. Cancer Sci. 2016;107(12):1818-1824.

25. Stein SM, James ES, Deng Y, et al. Final analysis of a phase II study of modified FOLFIRINOX in locally advanced and metastatic pancreatic cancer. Br J Cancer. 2016;114(7):737-743.

26. Wada K, Sano K, Amano H, et al. Biweekly gemcitabine plus S-1 for locally advanced and metastatic pancreatic cancer: a preliminary feasibility study. J Hepatobiliary Pancreat Sci. 2015;22(9):692-698.

27. Yamaue H, Satoi S, Kanbe T, et al. Phase II clinical study of alternateday oral therapy with S-1 as first-line chemotherapy for locally advanced and metastatic pancreatic cancer. Cancer Chemother Pharmacol. 2014; 73(1):97-102.

\section{Dovepress}

patient perspectives such as quality of life, adherence and satisfaction. The manuscript management system is completely online and includes a very quick and fair peer-review system, which is all easy to use. Visit http://www.dovepress.com/testimonials.php to read real quotes from published authors. 